\title{
SCIENTIFIC REPORTS

\section{OPEN Nucleus-specific linker histones Hhol and Mlh1 form distinct protein interactions during growth, starvation and development in Tetrahymena thermophila}

\author{
Syed Nabeel-Shah ${ }^{1,5,6,7}$, Kanwal Ashraf ${ }^{2,7}$, Alejandro Saettone ${ }^{1,7}$, Jyoti Garg ${ }^{2,7}$, \\ Joanna Derynck ${ }^{1}$, Jean-Philippe Lambert ${ }^{3,4}$, Ronald E. Pearlman ${ }^{2 *}$ \& Jeffrey Fillingham ${ }^{1 *}$
}

Chromatin organization influences most aspects of gene expression regulation. The linker histone $\mathrm{H} 1$, along with the core histones, is a key component of eukaryotic chromatin. Despite its critical roles in chromatin structure and function and gene regulation, studies regarding the $\mathrm{H} 1$ proteinprotein interaction networks, particularly outside of Opisthokonts, are limited. The nuclear dimorphic ciliate protozoan Tetrahymena thermophila encodes two distinct nucleus-specific linker histones, macronuclear Hho1 and micronuclear Mlh1. We used a comparative proteomics approach to identify the Hhol and Mlh1 protein-protein interaction networks in Tetrahymena during growth, starvation, and sexual development. Affinity purification followed by mass spectrometry analysis of the Hhol and Mlh1 proteins revealed a non-overlapping set of co-purifying proteins suggesting that Tetrahymena nucleus-specific linker histones are subject to distinct regulatory pathways. Furthermore, we found that linker histones interact with distinct proteins under the different stages of the Tetrahymena life cycle. Hhol and Mlh1 co-purified with several Tetrahymena-specific as well as conserved interacting partners involved in chromatin structure and function and other important cellular pathways. Our results suggest that nucleus-specific linker histones might be subject to nucleus-specific regulatory pathways and are dynamically regulated under different stages of the Tetrahymena life cycle.

The fundamental repeating unit of eukaryotic chromatin, the core nucleosome particle, is comprised of two of each of the core histones $\mathrm{H} 2 \mathrm{~A}, \mathrm{H} 2 \mathrm{~B}, \mathrm{H} 3$ and $\mathrm{H} 4$, and $\sim 147 \mathrm{bp}$ of $\mathrm{DNA}^{1}$. In addition to the core histones, there are linker histones ( $\mathrm{H} 1$ or $\mathrm{H} 5$ ) which flank the core nucleosomes sealing the structure with $20 \mathrm{bp}$ of additional $\mathrm{DNA}^{2}$. Within the past three decades, core histones have been extensively studied ${ }^{3}$, however, much less is known regarding the linker histones.

Different numbers of $\mathrm{H} 1$ variants have been identified across organisms ${ }^{4,5}$. For example, mammals contain 11 linker histone variants ${ }^{6}$ whereas Xenopus laevis and Caenorhabditis elegans have five and eight variants, respectively ${ }^{7}$. Most $\mathrm{H} 1$ variants are similar in their architecture such that they have an $\mathrm{N}$-terminal region, a C-terminal tail, and a central conserved globular region ${ }^{2}$. Saccharomyces cerevisiae linker histone Hholp is more divergent than its mammalian counterparts and contains two regions of sequence similarity to the central globular domain of the canonical histone $\mathrm{H}^{8}$. Histone $\mathrm{H} 1$ has been reported to function in stabilization of chromatin structure ${ }^{2}$, DNA replication ${ }^{9,10}$ as well as gene expression regulation ${ }^{11}$.

\footnotetext{
${ }^{1}$ Department of Chemistry and Biology, Ryerson University, 350 Victoria St., Toronto, M5B 2K3, Canada. ${ }^{2}$ Department of Biology, York University, 4700 Keele St., Toronto, M3J 1P3, Canada. ${ }^{3}$ Department of Molecular Medicine and Cancer Research Centre, Université Laval, Quebec, Canada. ${ }^{4} \mathrm{CHU}$ de Québec Research Center, CHUL, 2705 Laurier Boulevard, Quebec, G1V 4G2, Canada. ${ }^{5}$ Present address: Donnelly Centre, University of Toronto, Toronto, M5S 3E1, Canada. ${ }^{6}$ Department of Molecular Genetics, University of Toronto, Toronto, M5S 1A8, Canada. ${ }^{7}$ These authors contributed equally: Syed Nabeel-Shah, Kanwal Ashraf, Alejandro Saettone and Jyoti Garg. *email: ronp@yorku.ca; jeffrey.fillingham@ryerson.ca
} 
Linker histone $\mathrm{H} 1$ is known to carry posttranslational modifications (PTMs) ${ }^{11}$. Although the function of H1 PTMs is not well studied, several PTMs including phosphorylation, methylation, acetylation, citrullination, ubiquitylation, formylation, denitration, ADP-ribosylation, crotonylation, and lysine 2-hydroxyisobutyrylation have been identified ${ }^{2}$. Many of the enzymes regulating H1 PTMs are not known ${ }^{2,11}$. Eukaryotic gene expression is subjected to an ever-increasing list of regulatory layers ${ }^{3}$. Recently, metabolic enzymes have been recognized as regulators of various chromatin- and gene expression-related pathways ${ }^{12}$. Various metabolic enzymes, including glycolytic enzymes such as pyruvate kinase M2 isoform (PKM2), 6-phosphofructo-2-kinase/ fructose-2,6-bisphosphatase 4 (PFKFB4), fructose-1,6-bisphosphatase 1 (FBP1), glyceraldehyde-3-phosphate dehydrogenase (GAPDH), and tricarboxylic acid (TCA) cycle enzymes such as $\alpha$-ketoglutarate dehydrogenase $(\alpha-\mathrm{KGDH})$ and fumarase as well as enzymes involved in nucleotide synthesis such as inosine $5^{\prime}$-monophosphate dehydrogenase (IMPDH) and GMP synthase (GMPS), have been shown to localize to the nucleus where they may participate in chromatin regulation by modifying the histones and/or supplying metabolites necessary for histone or chromatin modifying enzymes ${ }^{12}$. For example, PKM2, which functions in glycolysis, was recently shown to directly bind with $\mathrm{H} 3$ to mediate phosphorylation at the threonine 11 residue ${ }^{13}$. Whether the role of metabolic enzymes in chromatin regulation is conserved across eukaryotes remains unclear.

The complexes depositing core histones and their variants on chromatin have been extensively studied ${ }^{3,14}$. For example, core histone H3.1 (or H3.2) is assembled onto chromatin only during S phase in a DNA replication dependent manner (RD) by a heterotrimeric CAF1 complex, whereas the variant H3.3 is deposited throughout the cell cycle in a replication independent (RI) fashion by the HIRA histone chaperone ${ }^{14-17}$. Furthermore, many of the generalized $\mathrm{H} 3 / \mathrm{H} 4$-specific histone chaperones, such as Asf1 and nuclear autoantigenic sperm protein (NASP), have been identified and extensively studied ${ }^{18}$. Like $\mathrm{H} 3$, the chromatin assembly of $\mathrm{H} 2 \mathrm{~A}$ and its variant H2A.Z (Htzl in yeast) is also tightly regulated in a cell cycle dependent manner via specialized chaperoning networks ${ }^{19}$. In contrast to the core histones, the deposition complexes of linker histones are not well characterized. Recently, it was reported that human linker histones associated with functionally diverse proteins including RNA-binding proteins, transcriptional regulators, as well as ribosomal proteins ${ }^{20}$. In addition to their roles in core histone metabolism, several proteins including nucleosome assembly protein 1 (Nap1) and NASP, are thought to function as $\mathrm{H} 1$ chaperones ${ }^{2,19,21-23}$, although mechanistic details remain largely unknown ${ }^{24}$.

Tetrahymena thermophila, a ciliate protozoan, is a well-suited model system to study chromatin biology and investigate gene expression regulatory layers ${ }^{25-27}$. Tetrahymena features two distinct nuclei, a macronucleus (MAC) and a micronucleus (MIC), present within the same cell. The polyploid MAC essentially controls all the transcription and divides amitotically during vegetative growth ${ }^{28}$. The diploid MIC is mostly transcriptionally silent, ensures stable inheritance of the genetic material and divides mitotically in vegetatively growing Tetrahymena. The two nuclei originate from the same zygotic nucleus during Tetrahymena sexual development (conjugation $)^{28}$. During Tetrahymena conjugation, extensive chromatin alterations take place in the developing nuclei including DNA rearrangements and removal of 'internally eliminated sequences (IES)' giving rise to progeny nuclei with distinct chromatin states ${ }^{29-31}$. Tetrahymena conjugation can be induced by starving the cells and mixing cells of two different mating types. Starvation in Tetrahymena is a physiological state that is known to induce numerous behavioral, phenotypic and molecular alterations making cells competent to embark on sexual development ${ }^{32,33}$.

The Tetrahymena genome encodes two linker histones with distinct nuclear localization ${ }^{34}$. The MAC-specific linker histone Hhol is thought to be a homolog of mammalian H1 but it lacks the conserved central globular domain ${ }^{35,36}$. Hho1 in Tetrahymena in not essential for cell viability and/or chromatin packaging ${ }^{34}$, however, it can regulate expression of certain genes by its differential phosphorylation ${ }^{37-39}$. Tetrahymena cells lacking the HHO1 gene appear to have enlarged MACs but normal MICs ${ }^{34}$. The MIC-specific linker histone 1 (Mlh1) is a much larger protein which is proteolytically processed into smaller fragments $(\alpha, \beta, \gamma$, and $\delta)$ after its transport into the $\mathrm{MIC}^{40,41}$. The $\alpha$ fragment represents the un-cleaved state of $\delta$ and $\gamma$ fragments. The $\beta$ and $\gamma$ fragments appear to resemble $\mathrm{H} 1$ without a globular domain whereas the $\delta$ fragments have two high mobility group (HMG) boxes. Like Hho1, Mlh1 is not essential for cell viability during vegetative growth. MLH1 knockout Tetrahymena cells display enlarged MICs but normal MACs ${ }^{34}$. The regulatory pathways ensuring the differential transport of nucleus-specific linker histones in Tetrahymena remain unknown. Recently, nucleus-specific localization signals (NLS) have been identified for Hhol and Mlh1 ${ }^{41}$. However, the protein factors responsible for the transport of linker histones to specific nuclei have not yet been identified.

Here we employed a comparative proteomic approach to identify the protein-protein interactions of Hho 1 and Mlh1 under three different physiological conditions; growth, starvation and conjugation. We carried out affinity purification combined with mass spectrometry (AP-MS) analysis of endogenously tagged Hho1-FZZ and Mlh1-FZZ proteins. Our results reveal that Hho1-FZZ and Mlh1-FZZ interact with distinct sets of proteins under different physiological conditions. Furthermore, we found that the Tetrahymena linker histones interact with non-overlapping proteins suggesting that the nucleus-specific linker histones have distinct regulatory pathways. We suggest that Tetrahymena linker histones are dynamically regulated under distinct physiological stages to ensure their faithful transport and deposition to the specific nuclei.

\section{Results}

Engineering endogenously tagged Hho1-FZZ cell lines. The Tetrahymena genome contains a single gene encoding MAC-specific linker histone $H H O 1$, the protein product of which shares similarity with the $\mathrm{C}$-terminal region of the metazoan $\mathrm{H} 1$ but lacks the central globular domain ${ }^{35,36}$. Our secondary structure prediction of Hhol indicated that its $\mathrm{N}$-terminus (residues 1-75) is composed mostly of helices whereas the remainder of the protein is largely disordered, consistent with the structure of the metazoan linker histone C-terminus ${ }^{42}$ (Fig. 1A). 


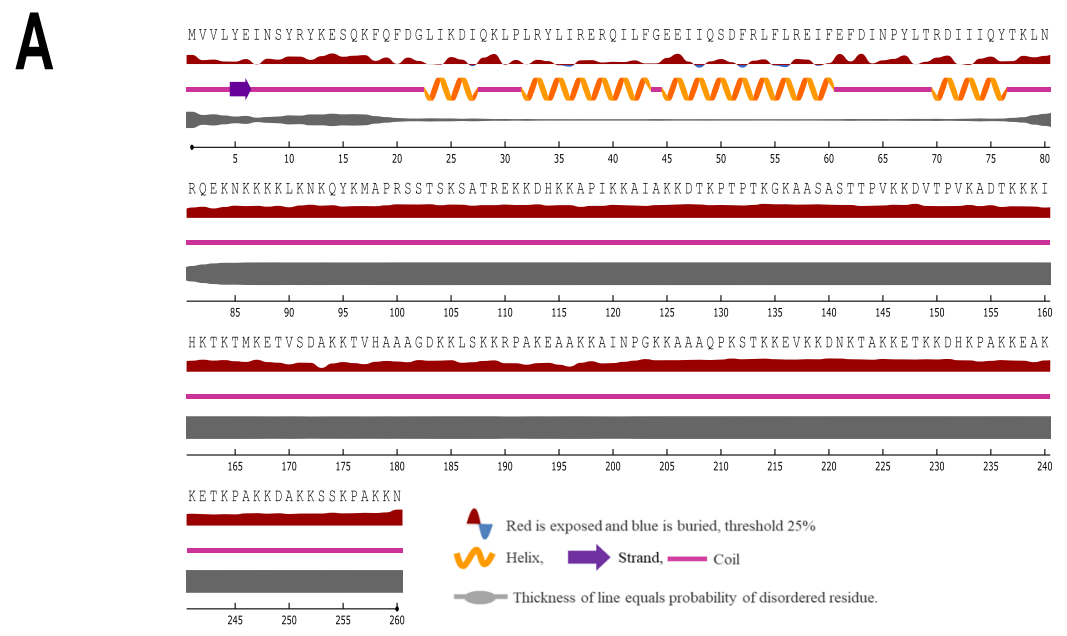

B
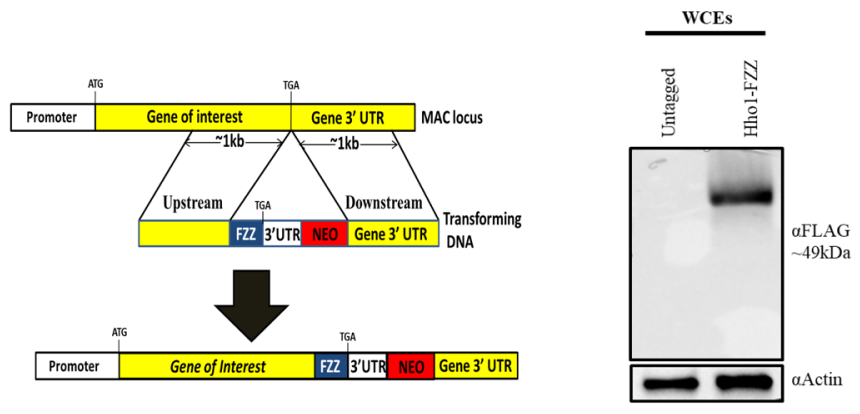

C
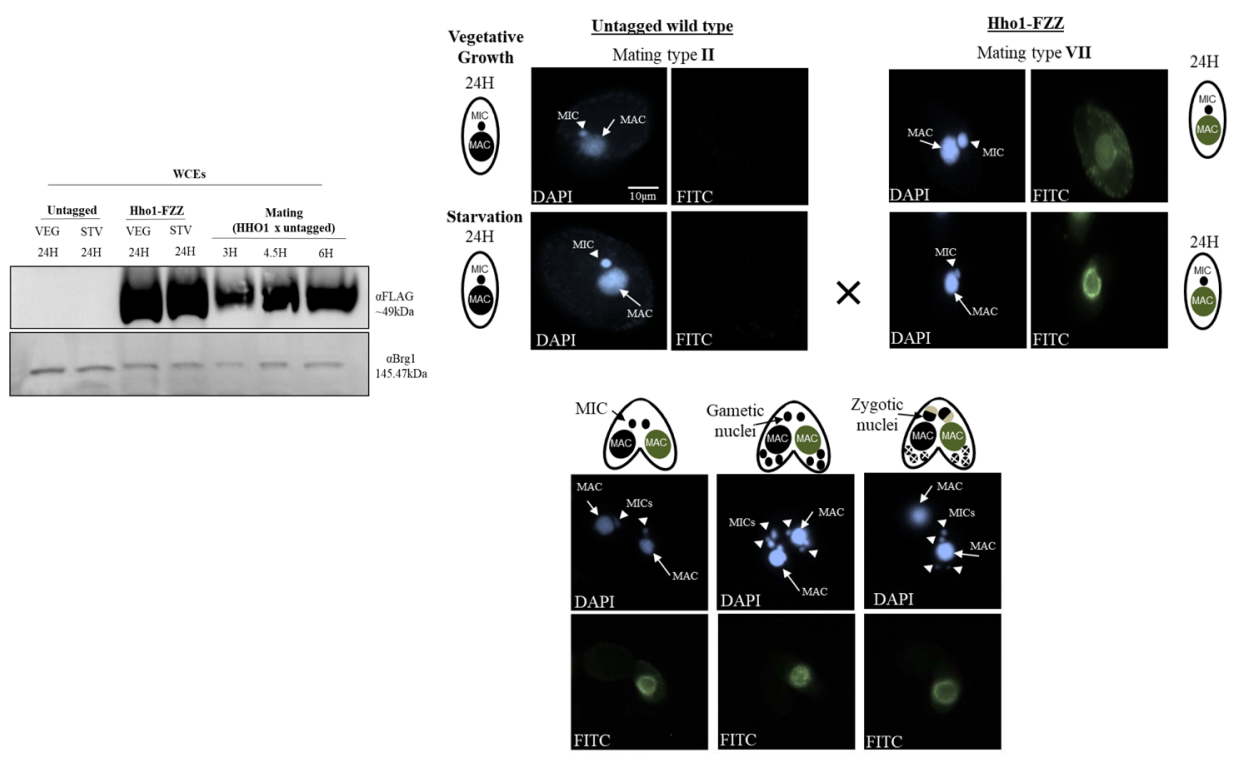

Figure 1. Hho1-FZZ expression and localization in the MAC. (A) Predicted secondary structure of Tetrahymena MAC-specific linker histone Hhol. Hhol protein is mostly composed of disordered regions. (B) Left- Schematic representation of epitope tagging strategy for the MAC locus. Right- Expression analysis of endogenously tagged Hhol-FZZ in comparison to the untagged controls by Western blotting in whole cell extracts (WCEs). Top panel was probed with anti-FLAG in order to detect Hhol-FZZ and the bottom panel was probed with anti-Actin as a loading control. (C) Left- Western blotting analysis using Hho1-FZZ WCEs to examine its expression across the indicated stages of the Tetrahymena life cycle. VEG-vegetative growth; STV-starvation; mating-conjugating Tetrahymena cells. Top panel was probed with anti-FLAG antibody to detect Hhol-FZZ and the bottom panel was probed with anti-Brg1 as a loading control. Right- HHol-FZZ localizes to the MAC only and not to the MIC during vegetative growth, starvation and conjugation. Hho1-FZZ cells of mating type VII were mated with the untagged cells of mating type II. Nuclear events are depicted as cartoons. DAPI was used to stain the nuclei. The images were processed using ImageJ (version 1.50i) (https://imagej.nih.gov/ij/). 
Toward our goal of identifying Hhol protein-protein interactions, we generated a Tetrahymena cell line stably expressing $\mathrm{HHO} 1$ with a C-terminal FZZ epitope tag from its endogenous MAC locus (Fig. 1B, left). We previously successfully employed the FZZ epitope tag, comprised of $3 \times$ FLAG and 2 protein A moieties separated by a TEV cleavage site, to perform affinity purification experiments and conduct indirect immunofluorescence (IF) studies ${ }^{25}$. The expression of endogenously tagged HHO1-FZZ was confirmed by Western blotting analysis in whole cell extracts (WCEs) prepared either from HHO1-FZZ expressing cells or untagged wild-type Tetrahymena (Fig. 1B, right). We next examined the expression levels of Hhol-FZZ during vegetative growth, starvation and conjugation 3-, 4.5- and 6-hours post mixing the different mating types. We observed that Hho1-FZZ is abundantly expressed throughout the examined stages of the Tetrahymena life cycle (Fig. 1C, left). To further examine the functionality of endogenously tagged Hho1-FZZ, we carried out IF analysis through the above stages of the Tetrahymena life cycle. Consistent with previous studies ${ }^{41}$, we found that Hhol-FZZ is exclusively localized to the MAC throughout the examined stages of the Tetrahymena life cycle (Fig. 1C, right), indicating that the FZZ tag does not interfere with the proper localization of Hhol-FZZ.

Interactome mapping of Hhol throughout Tetrahymena life cycle. To identify Hho1-FZZ protein-protein interactions (PPIs), we performed affinity purification (AP) coupled to mass spectrometry analysis (AP-MS). We adopted a comparative proteomics approach and examined Hhol interaction profiles through different stages of the Tetrahymena life cycle including growth, starvation (24hours) and development (6 hours post mixing, a time when most mating cells have finished post-zygotic nuclear divisions). The recovery of affinity purified bait was monitored by Western blotting (Fig. 2, top). We used 'Significance Analysis of INTeractome' (SAINTexpress) analysis to evaluate our MS data ${ }^{43}$. The SAINTexpress utilizes semiquantitative spectral counts for assigning a confidence value to individual PPIs ${ }^{43}$. We used untagged wildtype Tetrahymena cells for control purifications from the appropriate cell cycle stages (i.e., during growth, starvation or development). The Hho1-FZZ AP-MS data filtered against control purifications identified a set of interacting partners that passed the statistical threshold (Bayesian false discovery rate $(F D R) \leq 1 \%$ ) and are depicted in Fig. 2 (Supplemental File S1).

Hhol-FZZ co-purified with distinct interaction partners through the examined stages of the Tetrahymena life cycle (Fig. 2, bottom). The Hho1-FZZ co-purifying proteins were analyzed for domain architecture and possible similarities to budding yeast and/or human proteins. During vegetative growth Hho1-FZZ co-purified with 28 high-confidence (FDR $\leq 1 \%)$ interacting partners. Among the vegetative-specific Hho1-FZZ co-purifying proteins were 6 hypothetical proteins without any recognizable domains, two growth-arrest-specific 8 (GAS8) proteins, a tetratricopeptide (TPR) repeat domain protein, two putative protein kinases including Camk1 and Cmk2, ribosomal proteins Rpl19 and Rpl21, Ubp14 and the putative splicing factor Snp1 (U1 small nuclear ribonucleoprotein $70 \mathrm{kDa}$ homolog) (Fig. 2; see Supplemental File S1 for details and the full list of Hho1 PPIs). Additionally, we identified Jub6-like 1 (Jsl1 (TTHERM_01040830)) protein to interact with Hhol during vegetative growth at a slightly relaxed statistical threshold of BFDR $\leq 0.02$. Jsl1 is closely related to a prion-like protein Jub6 in Tetrahymena which has previously been shown to be involved in the regulation of chromatin structure ${ }^{44}$.

Application of SAINTexpress revealed three high-confidence Hho1-FZZ interaction partners in starving Tetrahymena cells. These include a coiled-coil domain protein, Jsl1, which was also identified in vegetative growth (see above), and Mxr1 (methionine-S-sulfoxide reductase 1). Mxr1 was also identified as a high-confidence Hho1-FZZ interacting partner during conjugation. The SAINTexpress analysis identified eight additional high-confidence Hhol-FZZ co-purifying proteins in conjugating Tetrahymena cells including TTHERM_00585170 which encodes a dense core granule associated C-terminal crystallin fold protein Ccf11, and two hypothetical proteins TTHERM_00105100 and TTHERM_00537380 both of which lack recognizable domains and appear to be Tetrahymena-specific without identifiable sequence similarity in any other organism. Based on sequence similarity to the budding yeast proteins, the remaining conjugation-specific Hho1-FZZ interaction partners include an acyl-CoA oxidase Pox1, rab-family GTPase Rab7 (or Ypt7), glycolytic enzyme Tpi1, peptidyl-tRNA hydrolase Pth1 and a metallopeptidase family M24 containing protein Fra1. Additionally, Jub6 was identified to co-purify with Hho1-FZZ during conjugation. It however did not pass our stringent statistical threshold of FDR $\leq 0.01$.

We used publicly available micro-array data ${ }^{45}$ to examine the expression profiles of Hho1-FZZ co-purifying proteins (Supplementary Fig. S1). We observed that Hho1-FZZ co-purifying partners have expression profiles that often correlated with the physiological state in which the interactions were detected. This suggests that Hhol and its interacting partners are functionally linked. It is important to note that the expression patterns do not always correlate with the interaction profiles ruling out the possibility of detecting false positive interactions in the AP-MS. For example, several of the Hho1-FZZ interaction partners detected during vegetative growth (e.g. CETP, GAS8 and MRNC9) have the highest expression levels either during starvation or conjugation (Supplementary Fig. S1).

Structural analysis of Mlh1 and of the high mobility group protein family in Tetrahymena. The Tetrahymena genome encodes a distinct MIC-specific linker histone gene MLH1. Mlh1 protein is more than twice the length of Hhol and contains two high-mobility group (HMG) boxes. The HMG domain is highly conserved throughout the eukaryotes and is known to function as chromatin architectural proteins ${ }^{46}$. Our structural analysis indicated that both HMG boxes in Mlh1 are composed of helices forming an 'L-shaped' fold, typical of the HMGs ${ }^{47}$ (Fig. 3A). Previous studies have found that the Mlh1 and another MIC-specific HMG-family protein, HmgB3, have similar expression profiles through Tetrahymena developmental stages, and that HmgB3 could functionally compensate for the loss of $M L H 1^{48}$. Both the Mlh1 and HmgB3, however, when knocked out individually are not essential for Tetrahymena growth suggesting that there might be additional functionally redundant protein ${ }^{48}$. We searched the Tetrahymena genome for HMGs and identified at least 11 genes encoding proteins with an HMG-box (Fig. 3B). To infer the genetic relationship and predict any functional similarities 

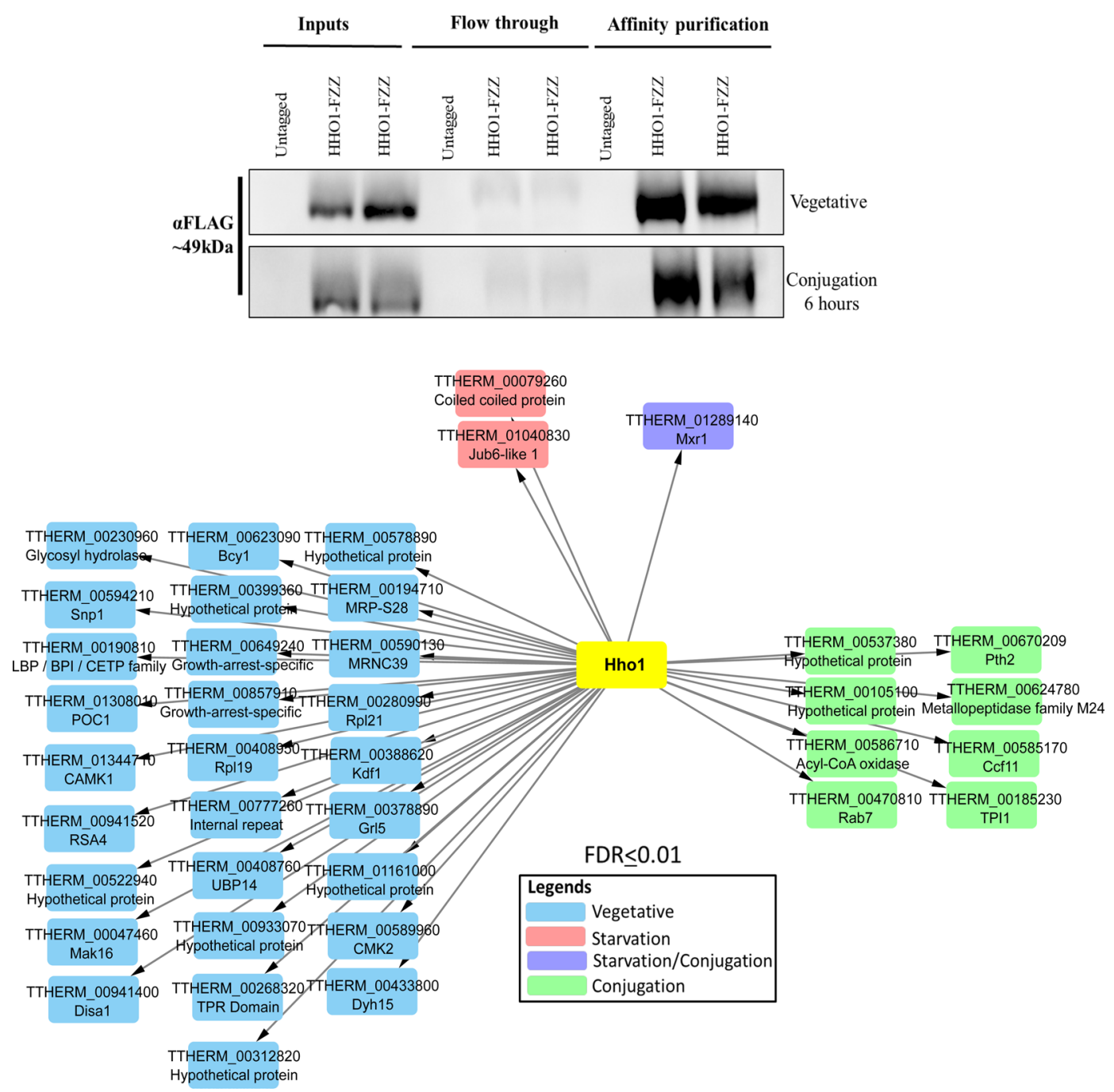

Figure 2. AP-MS analysis of Hho1-FZZ in three different physiological states of the Tetrahymena life cycle. Top: Western blotting analysis indicating recovery of the affinity purified Hho1-FZZ bait protein. The top panel represents the vegetative samples and the bottom panel shows the recovery of the bait from conjugation samples. The blots were probed with anti-FLAG antibody. No signal was detected in samples from the untagged control cells. Bottom: Network illustration of the Hho1-FZZ co-purifying proteins at statistical threshold of FDR $\leq 1 \%$ in three different stages of the Tetrahymena life cycle; vegetative growth, starvation (24hours) and conjugation (6 hours post mixing of the cells). Node color key is provided.

that might exist among the identified proteins, we carried out a phylogenetic analysis using the HMG-box amino acid sequences (Fig. 3C). Consistent with their proposed functional link ${ }^{48}$, HmgB3 and HMG-box 2 of Mlh1 clustered together. Another HMG-box protein HmgB6 (HMG-box 2) also clustered with HmgB3 and Mlh1, suggesting a functional link among these proteins. We analyzed the expression profiles of the identified Tetrahymena HMG-family proteins using publicly available micro-array data ${ }^{45}$ (Fig. 3D). Our analysis revealed that the expression of HMG-family proteins is temporally regulated during different stages of Tetrahymena development. Consistent with our phylogenetic analysis, HmgB6, HmgB3 and Mlh1 clustered together due to their very similar expression profiles (Fig. 3D), supporting the idea that, like $\mathrm{HmgB} 3$, HmgB6 might also be functionally redundant with Mlh1.

Functional proteomic analysis of Mlh1 during growth, starvation and development. We engineered a Tetrahymena cell line stably expressing MLH1 with a C-terminal FZZ epitope tag from its endogenous MAC locus. Western blotting analysis demonstrated the successful expression of endogenously tagged Mlh1-FZZ protein in WCEs prepared from the epitope-tagged strain (Fig. 4A). Consistent with previous studies ${ }^{40}$ (Fig. 4A; left), we found that full-length Mlh1-FZZ is not detectable due to its known proteolysis into four distinct fragments (Fig. 4A). To examine the subcellular localization of Mlh1-FZZ, we carried out IF analysis through different stages of the Tetrahymena life cycle including vegetative growth, starvation and sexual development. Consistent with previous studies ${ }^{41,49}$, our IF analysis indicated that Mlh1-FZZ exclusively localized to the MIC during Tetrahymena vegetative growth, starvation and conjugation stages examined (Fig. 4B). We also examined 


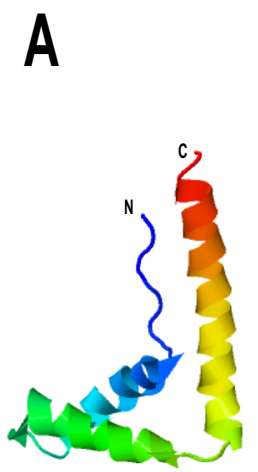

Predicted structure of HMG-box1 of Mlh1

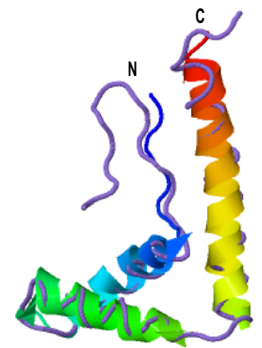

Superimposed with human Hmbg3 (PDB ID: 2YQI)

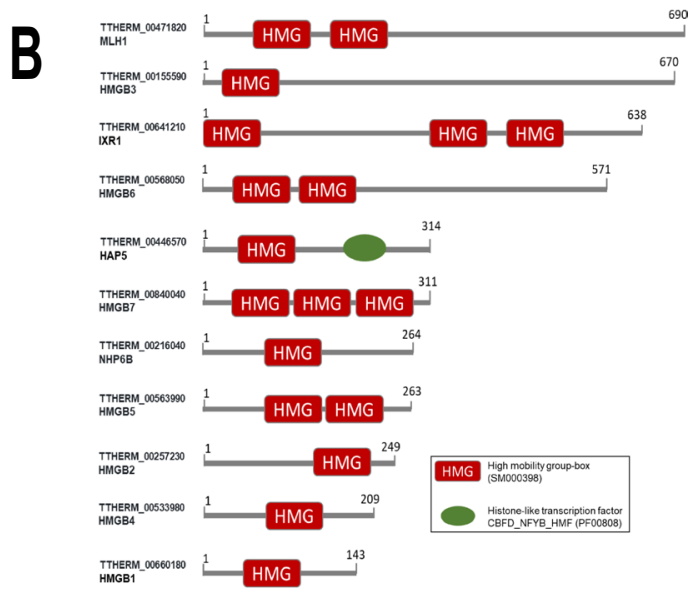

C

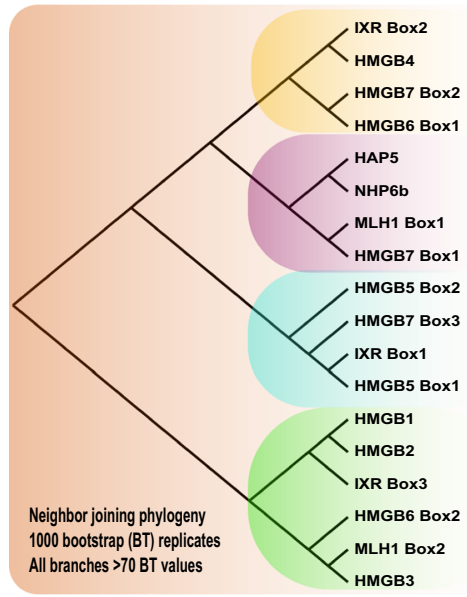

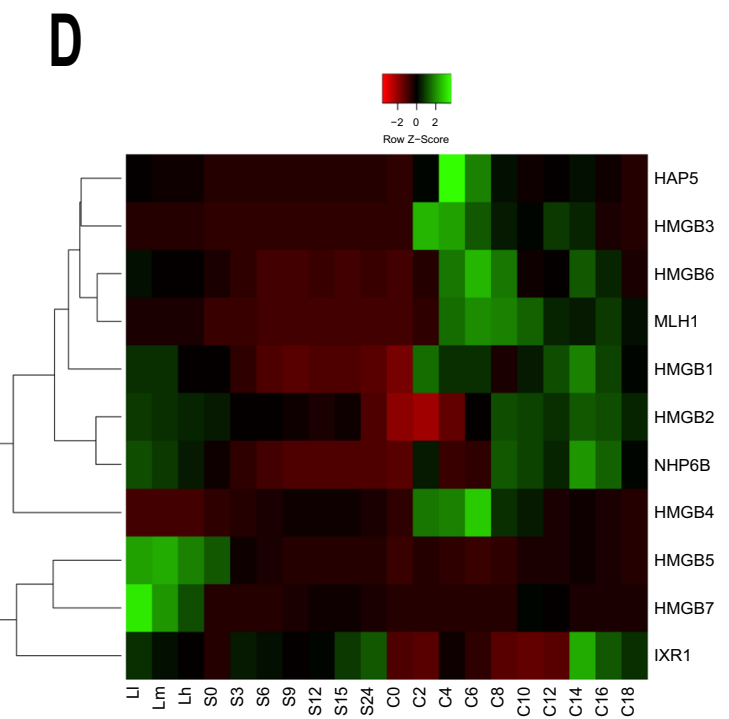

Figure 3. Mlh1 and HMG-box proteins in Tetrahymena. (A) Cartoon diagram shows the predicted structure of the Tetrahymena Mlh1 HMG-box 1 in rainbow color. The N- and C-termini are indicated. (B) Domain architecture of the HMG-family proteins identified in Tetrahymena. Domain legends are provided in the box. (C) Neighbour-joining phylogeny of HMG-box amino acid sequences from the identified HMG-family proteins in Tetrahymena. 1000 bootstrap replicates were performed to test the reliability of the resulting tree. All the nodes depicted had $>70$ bootstrap values. Node length does not indicate the genetic distance. (D) Heatmap represents the expression profiles based on hierarchical clustering of the microarray expression values for the HMG-family proteins across different stages of the Tetrahymena life cycle. Z-scores were calculated across each row to examine the differential expression across different stages. L1-LH: vegetative growth, S0-24: starvation for 24 hours, and C: conjugation (0-18 hours post mixing the cells of different mating types).

the Mlh1-FZZ localization in mitotically dividing MICs during vegetative growth and consistently observed a signal only in the MICs and not in the MACs (Fig. 4B). These results indicate that endogenously tagged Mlh1-FZZ is competent for proteolysis and nuclear import and that the presence of an FZZ tag does not alter its known localization profile.

To identify Mlh1-FZZ PPIs, we performed AP-MS experiments in biological replicates to examine Mlh1 interaction profiles through different stages of the Tetrahymena life cycle, including log-phase growth, starvation ( 24 hours) and development ( 6 hours post mixing, a time when most mating cells have finished post-zygotic nuclear divisions). Of note, previous work showed that the full-length Mlh1 is cleaved into four peptides upon its import to the $\mathrm{MIC}^{41}$. By WB (Fig. 4A), we found $\beta$-FZZ to be the major fragment observed suggesting that our AP-MS analysis captures the PPIs of the $\beta$-FZZ fragment. The full-length construct may also contribute to our MLH1 AP-MS results but to a lower degree. We used SAINTexpress analysis to evaluate our MS data ${ }^{43}$. The Mlh1-FZZ AP-MS data in biological replicates filtered against several control purifications yielded a set of 20 high-confidence co-purifying proteins (FDR $\leq 1 \%$ ) (Fig. 5A; Supplemental File S1) through three distinct Tetrahymena physiological conditions. 
A

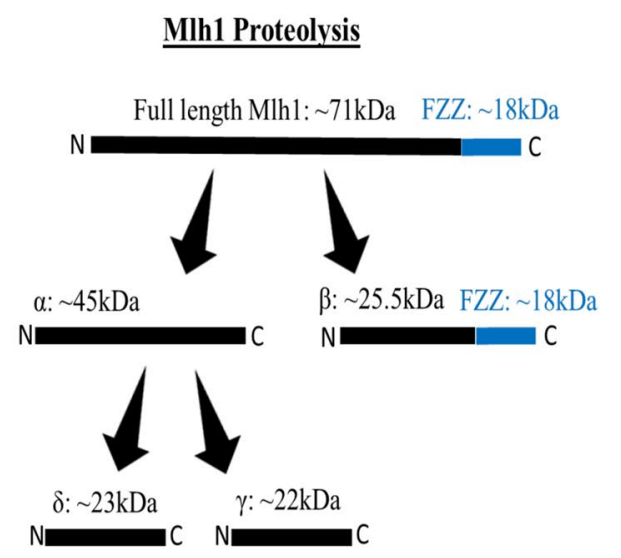

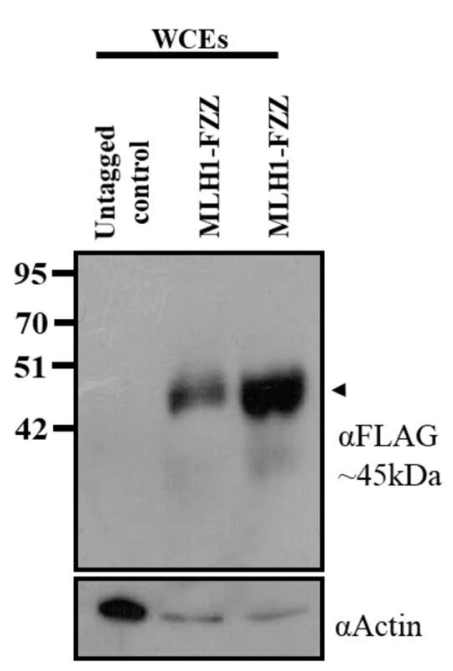

B
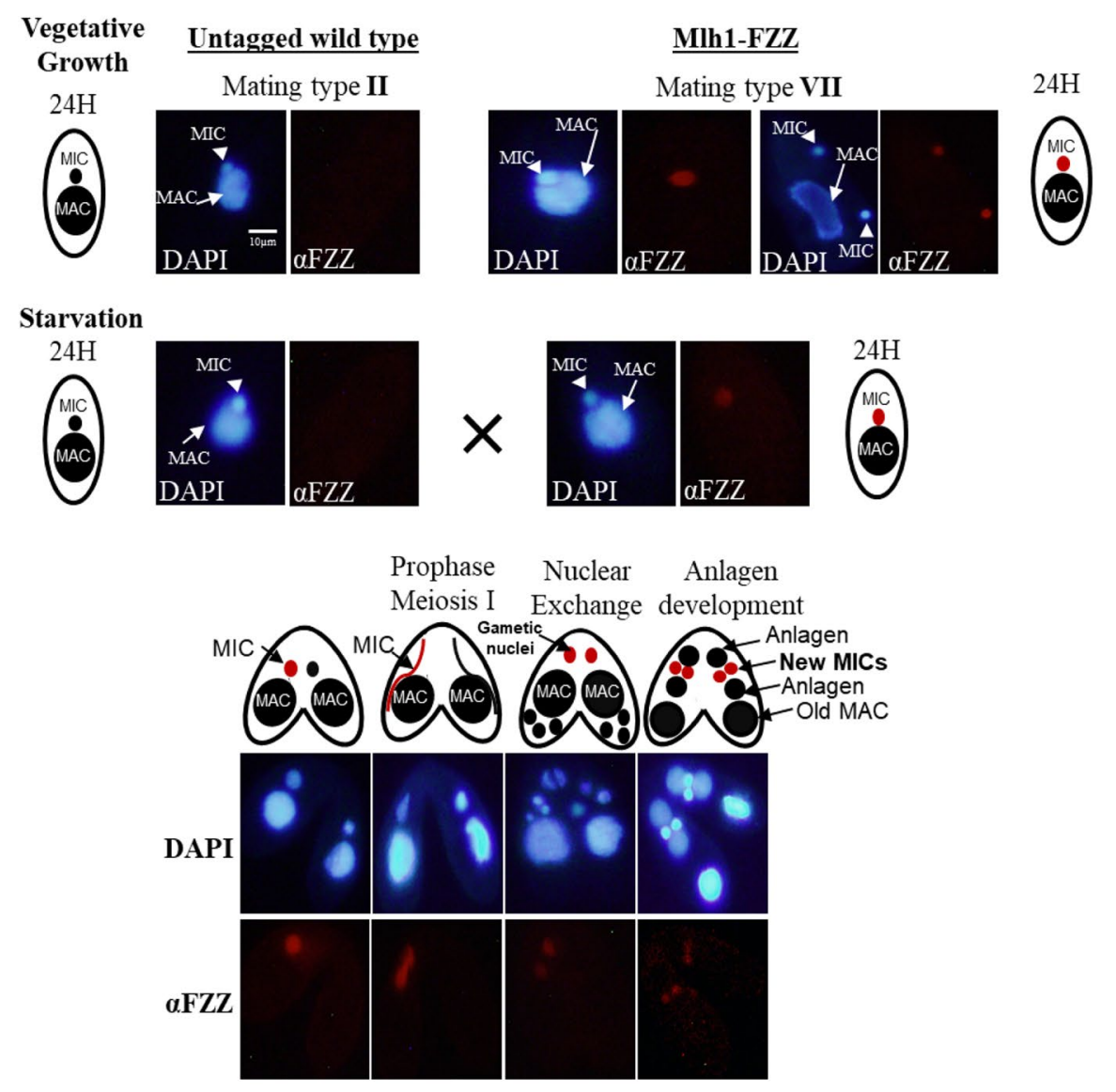

Figure 4. Mlh1-FZZ exclusively localizes to the MIC. (A) Left: Schematic representation of proteolytic cleavage of full length Mlh1. The predicted product size and molecular weights are indicated. The position and size of the FZZ tag is indicated in blue. Proteolysis of the full-length Mlh1-FZZ results in $\beta$-FZZ product. Right: Expression analysis of endogenously tagged Mlh1-FZZ in comparison to the untagged controls by Western blotting in WCEs. The black arrowhead represents the $\beta$ fragment $(\sim 25.5 \mathrm{kDa}+\mathrm{FZZ} 18 \mathrm{kDa})$. Anti-FLAG antibody was used to probe the top panel for Mlh1-FZZ detection. The bottom panel was probed with anti-Actin as a loading control. Two separate clones of Mlh1-FZZ were analysed. (B) Mlh1-FZZ localizes to MIC only during vegetative growth, starvation and conjugation. Mlh1-FZZ cells (mating type VII) were mated with untagged cells of mating type II. Nuclear events are illustrated as cartoons. Mlh1-FZZ signal was observed only in the MICs of the FZZ tagged cells and not in the untagged controls. The signal observed in both mating pairs (Mlh1-FZZ and controls) at the anlagen stage indicates mixing of cellular contents between the pairing cells. DAPI was used to stain the nuclei. The images were processed using ImageJ (version 1.50i) (https://imagej.nih.gov/ij/). 
A

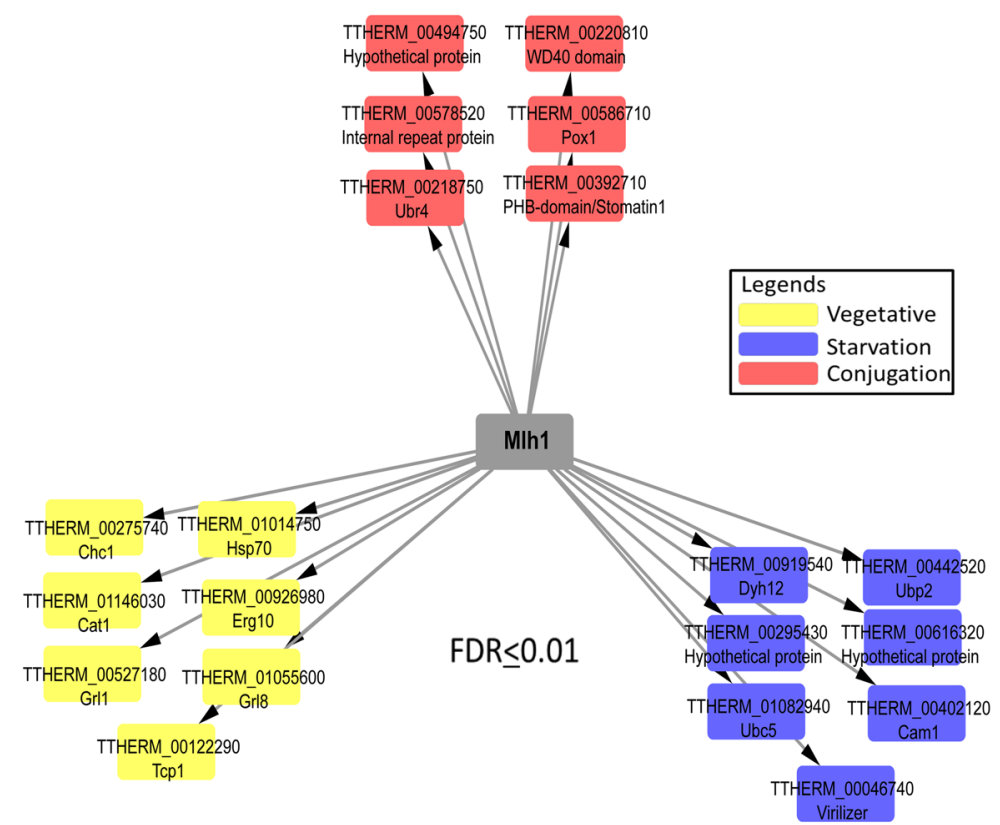

B

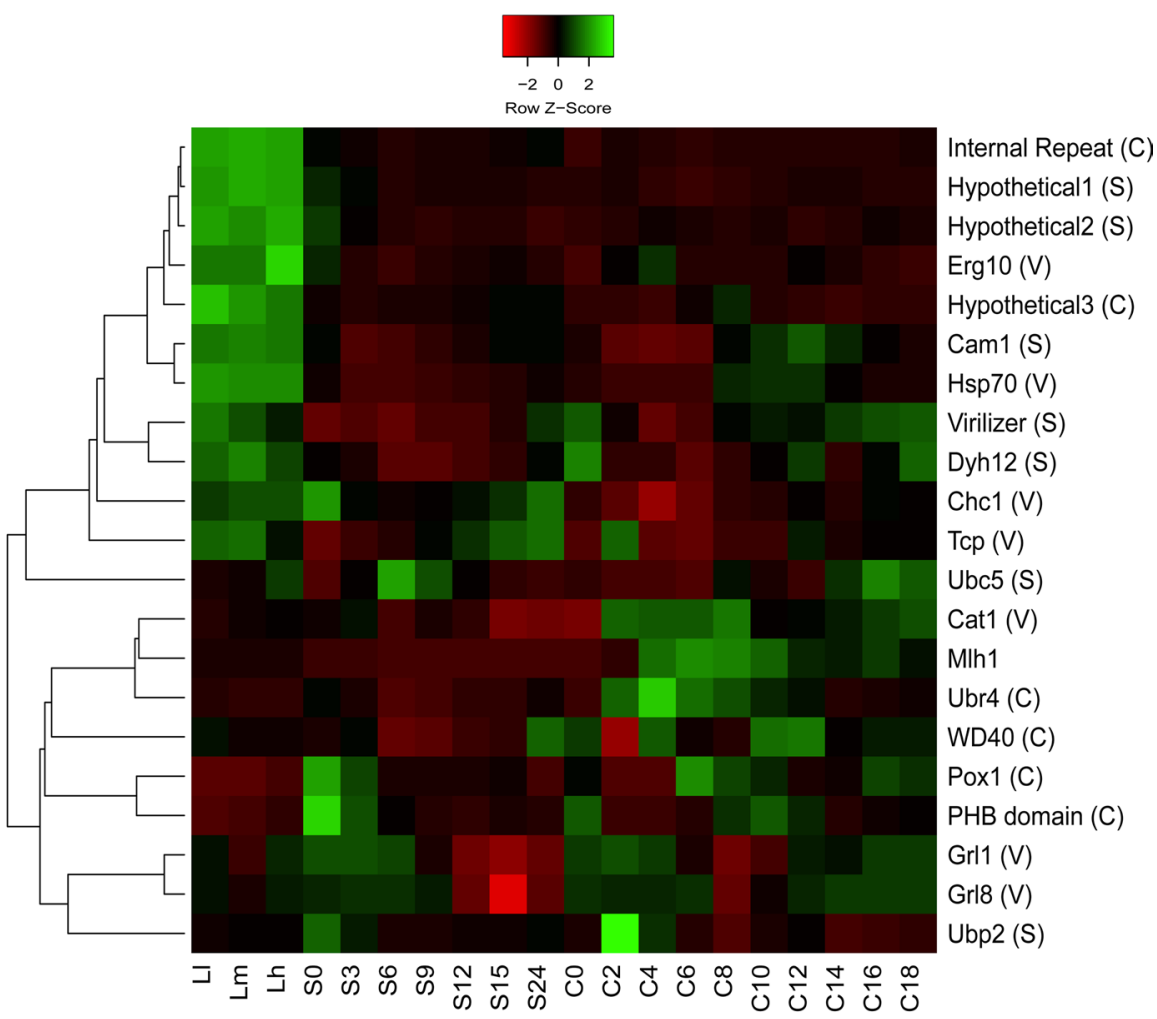

Figure 5. Mlh1-FZZ protein-protein interaction network. (A) A Network illustration of the Mlh1-FZZ copurifying proteins from three different stages of the Tetrahymena life cycle (statistical threshold of FDR $\leq 1 \%$ ). The node color legend is provided. (B) Heat map of microarray expression values for Mlh1-FZZ co-purifying proteins in the three different stages of the Tetrahymena life cycle. Z-scores were calculated across the rows for each protein to examine its differential expression across growth, starvation and various developmental stages. L1-LH: vegetative growth phase, S0-24: starvation for 24 hours, and C: conjugation where 0-18 denotes hours post mixing the different mating types. V, S and C in parenthesis after each prey protein name indicate vegetative, starvation and conjugation-specific interactions. 
During vegetative growth, Mlh1-FZZ co-purified with seven high-confidence binding partners including a heat shock protein Hsp70 and TCP-1/cpn60, both of which are thought to have a role in proper folding of other proteins $^{50,51}$ (Fig. 5A). Of note, the heat shock proteins including Hsp70 and Hsp90 have previously been shown to function in proper folding of newly synthesized histones $\mathrm{H} 3 / \mathrm{H} 4^{52}$. Additionally, SAINTexpress identified two Tetrahymena granule lattice proteins including Grl1 and Grl8, a peroxisomal enzyme Cat1, coat protein Chc1 and a putative homolog of budding yeast metabolic enzyme acetyl-CoA acyltransferase Erg10. Application of SAINTexpress to the Mlh1-FZZ AP-MS data from starving Tetrahymena cells revealed seven high-confidence interacting proteins including two hypothetical proteins, TTHERM_00295430 and TTHERM_00616320, both of which appear to be ciliate-specific and lack any recognizable domains. The SAINTexpress analysis identified TTHERM_00442520, TTHERM_01082940 and TTHERM_00402120 to copurify with Mlh1-FZZ in starving cells. These proteins share sequence similarities to budding yeast ubiquitin-specific protease Ubp2, ubiquitin-conjugating enzyme Ubc5 and nuclear protein Cam1, respectively. Although, ubiquitination of Tetrahymena Mlh1 is yet to be examined, co-purification of ubiquitin ligase suggests a role for this PTM in Mlh1 metabolism. Additionally, THERM_00046740 was identified as a high-confidence starvation-specific Mlh1-FZZ co-purifying protein which shares sequence similarity with human VIRMA (protein Virilizer homolog) protein and contains a signature VIR_N domain (Pfam accession: pf15912) (Supp. Fig. S2). In humans, VIRMA was recently shown to co-purify with linker histones ${ }^{20}$. In Drosophila melanogaster, Virilizer has been shown to be crucial for male and female viability and is required to produce eggs capable of embryonic development ${ }^{53}$.

Application of SAINTexpress revealed six high-confidence conjugation-specific ( 6 hours post mixing the different mating type Tetrahymena cells) Mlh1-FZZ interaction partners including hypothetical proteins TTHERM_00494750, TTHERM_00220810 and TTHERM_00578520. TTHERM_00494750 and TTHERM_00220810 contain internal repeats and a WD40 domain, respectively, whereas TTHERM_00578520 does not have any identifiable domains. The WD40 domain is thought to provide a scaffold to mediate protein-protein interactions and is found in many proteins with chromatin-related functions ${ }^{54}$. Additionally, SAINTexpress identified a PHB-domain containing protein TTHERM_00392710, a putative E3 ubiquitin ligase Ubr4 TTHERM_00218750 and a hypothetical protein TTHERM_00586710 which shares similarity to yeast fatty-acyl coenzyme A oxidase POX1 (Fig. 5A). Expression analysis using publicly available microarray data ${ }^{45}$ revealed that many of the conjugation-specific Mlh1-FZZ co-purifying proteins clustered together and their expression is increased between $4-8$ hours post-mixing of the cells (Fig. 5B).

\section{Discussion}

Linker histones are a key component of eukaryotic chromatin. Recent studies indicate that linker histone H1 functions in multiple chromatin-related processes including DNA replication ${ }^{9}$, DNA- repair ${ }^{55}$, and modulation of epigenetic information ${ }^{2}$. The information regarding the linker histone chaperones and deposition complexes outside of the Opisthokonts remains limited ${ }^{11,20}$. Here we report the proteomic analysis of Tetrahymena nucleus-specific linker histones Hhol and Mlh1. We utilized a comparative proteomic approach to profile the protein-protein interactions for the MAC-specific Hhol and MIC-specific Mlh1 through different stages of the Tetrahymena life cycle including growth, starvation and conjugation. We found that the nucleus-specific linker histones generally formed distinct non-overlapping protein-protein interactions under different physiological conditions.

We have previously reported the protein-protein interaction networks for the core histones $\mathrm{H} 2 \mathrm{~A}, \mathrm{H} 2 \mathrm{~B}$ and variant Hv1 in Tetrahymena ${ }^{25}$. The core histones H2A(Hv1)/H2B in Tetrahymena were found to interact with a highly conserved network of dedicated chaperones including the FACT-complex and nucleoplasmin1-like (cNPL1) protein ${ }^{25}$. Furthermore, we have previously shown that conserved histone chaperones Asf1 and NASP (Nrp1) likely function in $\mathrm{H} 3 / \mathrm{H} 4$ metabolism in Tetrahymena ${ }^{56,57}$, consistent with their known roles in other organisms ${ }^{14}$. We observed that the linker histones in Tetrahymena co-purify with functionally diverse as well as several lineage-specific proteins that lack orthologs in other organisms. This suggests that Tetrahymena linker histones might be subject to lineage-specific regulatory pathways. These observations are consistent with a recent proteomics analysis of human linker histone variants ${ }^{20}$. This study found that functionally diverse proteins including RNA-binding proteins, chromatin-related factors, ubiquitin specific peptidase as well as nucleolar proteins co-purify with linker histones ${ }^{20}$. Among the known human histone chaperones, Nap1L1 is thought to function as a linker histone chaperone in addition to its role as a core histone binding protein ${ }^{2,58,59}$. The Tetrahymena genome encodes TTHERM_00786930 which shares high sequence similarity with human and yeast Nap1 proteins. The Tetrahymena Nap1 did co-purify with Hhol in all three physiological conditions that we examined and with Mlh1 only during conjugation. It however did not pass our statistical threshold of FDR $\leq 1 \%$. Identifying the protein interaction network of Nap1 would provide insights into its possible functions in Tetrahymena. We suggest that unlike core histones with dedicated chaperoning networks that arose early during eukaryotic evolution ${ }^{14,25,26}$, the linker histones might be more flexible in terms of their interaction partners and may lack a set of conserved specialized chaperones.

Recent evidence suggests that histones are post-translationally modified by various metabolic enzymes, e.g. PKM2, PFKFB4, FBP1, GAPDH and $\alpha-K_{G D H}{ }^{12}$. Furthermore, linker histone $\mathrm{H} 1$ is also known to be ubiquitinated ${ }^{60,61}$. Our AP-MS data revealed that several putative metabolic enzymes co-purify with the linker histones (Supplementary Data S1; Figs. 2 and 5). This observation raises the possibility that Tetrahymena linker histones might also carry PTMs, such as acylation and ubiquitylation, although such epigenetic marks are yet to be examined in this model organism.

Jub6 protein forms prion-like aggregates and is a component of heterochromatin bodies in the developing MAC during Tetrahymena conjugation ${ }^{44}$. The Tetrahymena genome appears to encode at least six Jub6-like proteins (Jsl 1-6) ${ }^{44}$. These proteins however do not appear to have prion-like properties and their functions remain unknown ${ }^{44}$. Hho1 in Tetrahymena is differentially phosphorylated during vegetative growth and starvation ${ }^{37-39}$. 
The phosphorylation of Hhol has been shown to regulate transcription and mimics the loss of $H H O 1^{37}$. In starving Tetrahymena, Hhol is dephosphorylated which allows it to associate with chromatin and positively or negatively regulate the expression of certain genes ${ }^{37-39}$. Considering that $\mathrm{H} 1$ has been shown to be important for heterochromatin formation in other organisms ${ }^{62}$, and starvation in Tetrahymena features Hhol dependent compaction $^{37-39}$, it will be interesting to investigate the interplay between Hho1 and Jsl1.

Human VIRMA protein has recently been shown to co-purify with human linker histone variants ${ }^{20}$. The co-purification of a putative VIRMA ortholog with Mlh1 suggests a conserved role of VIRMA in linker histone metabolism. Recently, VIRMA was shown to function in mediating N6-methyladenosine (m6A) methylation of mRNAs as a component of the WMM complex ${ }^{63}$. In Drosophila melanogaster, Virilizer plays a crucial role in male and female viability and is thought to be required to produce eggs capable of embryonic development ${ }^{53}$. Starvation in Tetrahymena is known to induce distinct phenotypic alterations and molecular changes resulting in cellular competence for sexual development ${ }^{32,33}$. The co-purification of a putative VIRMA protein with Mlh1 in starving Tetrahymena cells suggests a possible role for it in preparing the cellular chromatin for sexual development.

In summary, our study presents the first profile of the Tetrahymena linker histone protein-protein interactions. Our results demonstrate that Tetrahymena MAC- and MIC-specific linker histones form distinct protein interaction networks under different physiological conditions. Carrying out BioID-MS ${ }^{64}$, an orthogonal approach to AP-MS, will be instrumental to provide a more detailed view of Mlh1 and Hhol interaction networks.

\section{Materials and Methods}

Cell strains and culture conditions. Tetrahymena inbreeding line B strains CU428 [Mpr/Mpr (VII, mp-s)] and B2086 [Mpr+/Mpr+ (II, mp-s)] were acquired from the Tetrahymena Stock Center, Cornell University, Ithaca N.Y. (http://tetrahymena.vet.cornell.edu/). Cells were cultured in $1 \times$ SPP media and were maintained axenically at $30^{\circ} \mathrm{C}$ as previously described ${ }^{65}$. To induce conjugation, exponentially growing Tetrahymena cells of two different mating types were starved overnight at $30^{\circ} \mathrm{C}$ without shaking in $10 \mathrm{mM}$ Tris- $\mathrm{HCl} \mathrm{pH} \mathrm{7.5.} \mathrm{The} \mathrm{starved} \mathrm{cells} \mathrm{were}$ mixed in roughly equal numbers $\left(5 \times 10^{5}-7 \times 10^{5}\right.$ cells $\left./ \mathrm{mL}\right)$ to allow conjugation at $30^{\circ} \mathrm{C}$ without shaking.

Macronuclear gene replacement. To construct the epitope tagging vectors for $H H O 1$ and $M L H 1, \sim 1 \mathrm{~kb}$ DNA fragments upstream and downstream of the predicted stop codons of each gene were amplified by standard PCR reactions. The PCR products were digested with KpnI/XhoI and NotI/SacI for the upstream and downstream fragments, respectively. The digested products were cloned into the appropriate sites within the tagging vector (pBKS-FZZ) provided by Dr. Kathleen Collins (University of California, Berkeley, CA). The final plasmid was double digested with KpnI and SacI for linearization prior to biolistic transformation into Tetrahymena cells. We used one micrometer gold particles $(60 \mathrm{mg} / \mathrm{mL}$; Bio-Rad) and coated them with $5 \mu \mathrm{g}$ of the linearized plasmid DNA. The gold particles were introduced into the Tetrahymena MAC via biolistic transformation with a PDS-1000/He Biolistic particle delivery system (Bio-Rad). The transformants were selected using paromomycin $(60 \mu \mathrm{g} / \mathrm{mL})$. MAC homozygosity was achieved by growing the cells in increasing concentrations of paromomycin to a final concentration of $1 \mathrm{mg} / \mathrm{ml}$.

Generation of whole cell extracts (WCE) and Western blotting. $10 \%$ trichloroacetic acid was used to prepare WCE by incubation on ice for 30 minutes. The WCEs were re-suspended in $500 \mu \mathrm{L}$ of SDS loading dye. $10 \mu \mathrm{L}$ of $1 \mathrm{~N} \mathrm{NaOH}$ was added to neutralize the final solution, if required. WCEs were subjected to electrophoresis through 10\% SDS-PAGE. Proteins transferred to a nitrocellulose membrane were blocked in 5\% skim milk and probed with the indicated antibodies. Antibodies and dilutions used were anti-Flag (1:4000; Sigma), anti-Actin (1:10000; Abcam), anti-Brg1 (1:1000, as described $\left.{ }^{66}\right)$

Affinity purification and mass spectrometry sample preparation. Affinity purification was carried out using our previously described method ${ }^{25,56}$. Briefly, Tetrahymena were grown in $\sim 500 \mathrm{~mL}$ of $1 \times$ SPP to a final concentration of $3 \times 10^{5}$ cells $/ \mathrm{mL}$ and were pelleted. For starvation, cells were washed once with $1 \times \mathrm{PBS}$, resuspended in $10 \mathrm{mM}$ Tris- $\mathrm{HCl} \mathrm{pH} 7.5$ and incubated at $30^{\circ} \mathrm{C}$ for 24 hours without shaking before pelleting. To induce conjugation, 24 hours starved cells of different mating types were mixed in equal numbers and incubated at $30^{\circ} \mathrm{C}$ for 24 hours without shaking. After 6 hours post mixing, cells were pelleted. The cell pellets for each of the above conditions were frozen at $-80^{\circ} \mathrm{C}$ until further use. The pellets were thawed on ice and re-suspended in lysis buffer $(10 \mathrm{mM}$ Tris- $\mathrm{HCl} \mathrm{pH} \mathrm{7.5,1} \mathrm{mM} \mathrm{MgCl}, 300 \mathrm{mM} \mathrm{NaCl}$ and $0.2 \% \mathrm{NP} 40$ plus yeast protease inhibitors (Sigma)). To eliminate the contaminating chromatin and/or RNA, Benzonase (Sigma E8263) nuclease was added (500 units) and extracts were rotated for 30 minutes at $4^{\circ} \mathrm{C}$. WCEs were clarified by centrifugation at $16,000 \mathrm{~g}$ for 30 minutes, and resulting soluble material was incubated with $50 \mu \mathrm{L}$ of packed M2-agarose (Sigma) at $4{ }^{\circ} \mathrm{C}$ for 3-4 hours. The M2-agarose was washed once with $10 \mathrm{~mL}$ IPP300 (10 mM Tris- $\mathrm{HCl} \mathrm{pH} 8.0,300 \mathrm{mM} \mathrm{NaCl}, 0.1 \%$ NP40), two times with $5 \mathrm{~mL}$ of IP100 buffer (10 mM Tris- $\mathrm{HCl} \mathrm{pH} \mathrm{8.0,100} \mathrm{mM} \mathrm{NaCl}, 0.1 \% \mathrm{NP} 40)$, and two times with $5 \mathrm{~mL}$ of IP100 buffer without detergent (10 mM Tris- $\mathrm{HCl} \mathrm{pH} \mathrm{8.0,100} \mathrm{mM} \mathrm{NaCl).} 500 \mathrm{uL}$ of $0.5 \mathrm{M} \mathrm{NH}_{4} \mathrm{OH}$ was used to elute the proteins by rotating the samples at room temperature for $\sim 20$ minutes.

Experimental design for mass spectrometry experiments. Two biological replicates were employed for each bait under study and processed independently. The samples were analysed alongside negative controls in each batch processed. For negative controls, we used untagged Tetrahymena cells (i.e. empty cells). In order to minimize the carry-over, extensive washes were performed between each sample (see details for each instrument type); and the order of sample acquisition on the mass spectrometer was reversed for the second replicate to circumvent systematic bias. 
Mass spectrometry sample preparation. Preparation of protein eluates for mass spectrometry acquisition was essentially as previously described ${ }^{67}$. Briefly, the eluates were dried using a speed vacuum apparatus and re-suspended in $10 \mu \mathrm{L}$ of $20 \mathrm{mM}$ Tris- $\mathrm{HCl} \mathrm{pH} \mathrm{8.0.} \mathrm{Trypsin} \mathrm{digestion} \mathrm{was} \mathrm{carried} \mathrm{out} \mathrm{using} 0.75 \mu \mathrm{g}$ of trypsin (Sigma) for $\sim 15$ hours at $37^{\circ} \mathrm{C}$ with mild agitation. An extra $0.25 \mu \mathrm{g}$ of trypsin was added to each sample and they were incubated for an additional 3 hours. The samples were acidified to a final concentration of $2 \%$ acetic acid, desalted using $\mathrm{C}_{18}$ StageTips (Thermo Scientific) as per the manufacturer's instructions and stored at $-80^{\circ} \mathrm{C}$ until their acquisition on a mass spectrometer.

Mass spectrometry acquisition using a Triple TOF 5600 mass spectrometer. $\quad 5 \mu \mathrm{L}$ of each sample, representing $50 \%$ of the sample, was directly loaded at $300 \mathrm{~nL} / \mathrm{min}$ onto a New Objective PicoFrit column $\left(15 \mathrm{~cm} \times 0.075 \mathrm{~mm}\right.$ I.D; Scientific Instrument Services, Ringoes, NJ) packed with Jupiter $5 \mu \mathrm{m} \mathrm{C}_{18}$ (Phenomenex, Torrance, CA) stationary phase. The peptides were eluted from the column by a gradient generated by an Agilent 1200 HPLC system (Agilent, Santa Clara, CA) equipped with a nano electrospray ion source coupled to a $5600+$ Triple TOF mass spectrometer (Sciex, Concord, ON). A 65-min linear gradient of a 5-35\% mixture of $0.1 \%$ formic acid injected at $300 \mathrm{~nL} / \mathrm{min}$ was used to elute peptides. Data dependent acquisition mode was used in Analyst version 1.7 (Sciex) to acquire mass spectra. Full scan mass spectra ( 400 to $1250 \mathrm{~m} / \mathrm{z}$ ) were acquired and followed by collision-induced dissociation of the twenty most intense ions. A period of 20 seconds and a tolerance of $100 \mathrm{ppm}$ were set for dynamic exclusion.

Data dependent acquisition MS analysis. Data dependent acquisition MS analysis was performed essentially as per ${ }^{56,67}$ with minor modifications. Mass spectrometry data were stored, searched and analyzed using the ProHits laboratory information management system (LIMS) platform ${ }^{68}$. Within ProHits, AB SCIEX WIFF files were first converted to an MGF format using WIFF2MGF converter and to an mzML format using ProteoWizard (v3.0.4468) and the AB SCIEX MS Data Converter (V1.3 beta). The mzML and mzXML files were searched using Mascot (v2.3.02). The spectra were searched with the RefSeq database (version45, January 24th, 2011) acquired from NCBI against a total of 24,770 T. thermophila sequences. For Triple TOF files, the database parameters were set to search for tryptic cleavages, allowing up to two missed cleavage sites per peptide with a mass tolerance of $40 \mathrm{ppm}$ for precursors with charges of $2+$ to $4+$ and a tolerance of $+/-0.15$ amu for fragment ions. Deamidated asparagine and glutamine and oxidized methionine were allowed as variable modifications. SAINTexpress version $3.61^{43}$ was used as a statistical tool to calculate the probability value of each potential protein-protein interaction from background contaminants using default parameters.

MS data visualization and archiving. We used Cytoscape (V3.4.0) $)^{69}$ to generate protein interaction networks. Individual nodes were manually arranged based on the physiological condition examined. The annotation of the co-purifying partners was carried out using BLAST searches and SMART domain analysis (http:// smart.embl-heidelberg.de $/)^{70}$ of the predicted amino acid sequences which were acquired from the Tetrahymena genome database (www.ciliate.org). All MS files used in this study were deposited at MassIVE (http://massive. ucsd.edu), assigned the MassIVE accession numbers MSV000084066. Additional details (including Mass IVE accession numbers and FTP download links) can be found in Supplemental File S1.

Identification and in silico analyses of HMG-proteins in Tetrahymena. We used the amino acid sequences of HMG-boxes present within Tetrahymena Mlh1 to search against the Tetrahymena genome database (www.ciliate.org). The proteins identified were analyzed by SMART domain analysis (http:// smart.embl-heidelberg.de $/)^{70}$ for the presence of HMGs. Phylogenetic analysis was carried out using the neighbor-joining method with 1000 bootstrap replicates. Amino acid sequences of the HMG-boxes from the identified proteins were used for the phylogeny reconstruction using MEGA7 ${ }^{71}$. For gene expression analysis, microarray data (accession number GSE11300) was acquired (http://tfgd.ihb.ac.cn/) and the expression values were represented in the heatmap format. Z-scores were calculated across each row to examine the differential expression across different stages of the Tetrahymena life cycle. We used hierarchical clustering to examine the similarities in gene expression profiles. Mlh1 structural predictions were carried out using the I-TASSER server ${ }^{72}$. Hhol secondary structure was predicted using PSIPRED ${ }^{73}$.

Indirect immunofluorescence. Tetrahymena cells were grown and fixed during vegetative growth, after 24 hours starvation, and 2, 4, 6 and 7 hours post mixing cells of different mating types to perform indirect immunofluorescence. The IF analysis was performed essentially as previously described ${ }^{56}$. Briefly, cells were washed in $10 \mathrm{mM}$ Tris- $\mathrm{HCl}, \mathrm{pH} 7.7$, and fixed in $4 \%$ paraformaldehyde. The cells were membrane-permeabilized with cold acetone for 20 minutes. Incubation with primary mouse anti-FLAG antibody (or anti-IgG (depicted as anti-FZZ)) (Sigma) was at a 1:500 dilution at $4^{\circ} \mathrm{C}$ overnight in $1 \times$ PBST. Cells were washed three times with $1 \times$ PBS before incubation in secondary antibody fluorescein isothiocyanate-conjugated (FITC) goat anti-mouse (Pierce) for 1 hour at room temperature. For nuclear counterstaining 4,6-diamidino-2-phenylindole dihydrochloride (DAPI) was used. Immunofluorescence analysis was carried out using an Olympus, DP70 equipped with a fluorescent microscope (Reichert-Jung, POLYVER) at 100x magnification and no oil was used. Final image preparation was carried out using ImageJ (version 1.50i) software (https://imagej.nih.gov/ij/ $/)^{74}$.

Received: 19 July 2019; Accepted: 14 November 2019;

Published online: 13 January 2020 


\section{References}

1. Luger, K., Mäder, A. W., Richmond, R. K., Sargent, D. F. \& Richmond, T. J. Crystal structure of the nucleosome core particle at 2.8 A resolution. Nature 389, 251-60 (1997).

2. Fyodorov, D. V., Zhou, B.-R., Skoultchi, A. I. \& Bai, Y. Emerging roles of linker histones in regulating chromatin structure and function. Nat. Rev. Mol. Cell Biol. 19, 192-206 (2018).

3. Mendiratta, S., Gatto, A. \& Almouzni, G. Histone supply: Multitiered regulation ensures chromatin dynamics throughout the cell cycle. J. Cell Biol. 39-54, https://doi.org/10.1083/jcb.201807179 (2018).

4. Biterge, B. \& Schneider, R. Histone variants: key players of chromatin. Cell Tissue Res. 356, 457-66 (2014).

5. Ponte, I., Romero, D., Yero, D., Suau, P. \& Roque, A. Complex Evolutionary History of the Mammalian Histone H1.1-H1.5 Gene Family. Mol. Biol. Evol. 34, 545-558 (2017)

6. Pan, C. \& Fan, Y. Role of H1 linker histones in mammalian development and stem cell differentiation. Biochim. Biophys. Acta 1859, 496-509 (2016).

7. Izzo, A., Kamieniarz, K. \& Schneider, R. The histone H1 family: specific members, specific functions? Biol. Chem. 389, 333-43 (2008).

8. Patterton, H. G., Landel, C. C., Landsman, D., Peterson, C. L. \& Simpson, R. T. The Biochemical and Phenotypic Characterization of Hholp, the Putative Linker Histone H1 of Saccharomyces cerevisiae. J. Biol. Chem. 273, 7268-7276 (1998).

9. Flickinger, R. A. Possible role of H1 histone in replication timing. Dev. Growth Differ. 57, 1-9 (2015).

10. Andreyeva, E. N. et al. Regulatory functions and chromatin loading dynamics of linker histone H1 during endoreplication in Drosophila. Genes Dev. 31, 603-616 (2017).

11. Hergeth, S. P. \& Schneider, R. The H1 linker histones: multifunctional proteins beyond the nucleosomal core particle. EMBO Rep. 16, 1439-53 (2015).

12. Li, X., Egervari, G., Wang, Y., Berger, S. L. \& Lu, Z. Regulation of chromatin and gene expression by metabolic enzymes and metabolites. Nat. Rev. Mol. Cell Biol. 19, 563-578 (2018).

13. Yang, W. et al. PKM2 phosphorylates histone H3 and promotes gene transcription and tumorigenesis. Cell 150, 685-96 (2012).

14. Grover, P., Asa, J. S. \& Campos, E. I. H3-H4 Histone Chaperone Pathways. Annu. Rev. Genet. 52, 109-130 (2018).

15. Tagami, H., Ray-Gallet, D., Almouzni, G. \& Nakatani, Y. Histone H3.1 and H3.3 complexes mediate nucleosome assembly pathways dependent or independent of DNA synthesis. Cell 116, 51-61 (2004).

16. Ray-Gallet, D. et al. HIRA is critical for a nucleosome assembly pathway independent of DNA synthesis. Mol. Cell 9, 1091-100 (2002).

17. Ray-Gallet, D. et al. Dynamics of histone $\mathrm{H} 3$ deposition in vivo reveal a nucleosome gap-filling mechanism for $\mathrm{H} 3.3$ to maintain chromatin integrity. Mol. Cell 44, 928-41 (2011).

18. Pardal, A. J., Fernandes-Duarte, F. \& Bowman, A. J. The histone chaperoning pathway: from ribosome to nucleosome. Essays Biochem. 63, 29-43 (2019).

19. De Koning, L., Corpet, A., Haber, J. E. \& Almouzni, G. Histone chaperones: an escort network regulating histone traffic. Nat. Struct. Mol. Biol. 14, 997-1007 (2007).

20. Zhang, P., Branson, O. E., Freitas, M. A. \& Parthun, M. R. Identification of replication-dependent and replication-independent linker histone complexes: Tpr specifically promotes replication-dependent linker histone stability. BMC Biochem. 17, 18 (2016).

21. Richardson, R. T. et al. Characterization of the histone H1-binding protein, NASP, as a cell cycle-regulated somatic protein. J. Biol. Chem. 275, 30378-30386 (2000).

22. Wang, H., Walsh, S. T. R. \& Parthun, M. R. Expanded binding specificity of the human histone chaperone NASP. Nucleic Acids Res. 36, 5763-5772 (2008).

23. Wang, H., Ge, Z., Walsh, S. T. R. \& Parthun, M. R. The human histone chaperone sNASP interacts with linker and core histones through distinct mechanisms. Nucleic Acids Res. 40, 660-669 (2012).

24. Harshman, S. W., Young, N. L., Parthun, M. R. \& Freitas, M. A. H1 histones: current perspectives and challenges. Nucleic Acids Res. 41, 9593-609 (2013).

25. Ashraf, K. et al. Proteomic Analysis of Histones H2A/H2B and Variant Hv1 in Tetrahymena thermophila Reveals an Ancient Network of Chaperones. Mol. Biol. Evol. 36, 1037-1055 (2019).

26. Saettone, A. et al. Functional Proteomics of Nuclear Proteins in Tetrahymena thermophila: A Review. Genes (Basel). 10, (333 (2019).

27. Garg, J. et al. The Med31 Conserved Component of the Divergent Mediator Complex in Tetrahymena thermophila Participates in Developmental Regulation. Curr. Biol., https://doi.org/10.1016/j.cub.2019.06.052 (2019).

28. Martindale, D. W., Allis, C. D. \& Bruns, P. J. Conjugation in Tetrahymena thermophila. A temporal analysis of cytological stages. Exp. Cell Res. 140, 227-36 (1982).

29. Yao, M.-C. C., Choi, J., Yokoyama, S., Austerberry, C. F. \& Yao, C.-H. H. DNA elimination in Tetrahymena: a developmental process involving extensive breakage and rejoining of DNA at defined sites. Cell 36, 433-40 (1984).

30. Yao, M.-C., Fuller, P. \& Xi, X. Programmed DNA deletion as an RNA-guided system of genome defense. Science 300, 1581-4 (2003).

31. Mochizuki, K. \& Gorovsky, M. A. RNA polymerase II localizes in Tetrahymena thermophila meiotic micronuclei when micronuclear transcription associated with genome rearrangement occurs. Eukaryot. Cell 3, 1233-40 (2004).

32. Bruns, P. J. \& Brussard, T. B. Pair formation inTetrahymena pyriformis, an inducible developmental system. J. Exp. Zool. 188, 337-344 (1974)

33. Bruns, P. J. \& Palestine, R. F. Costimulation in Tetrahymena pyriformis: a developmental interaction between specially prepared cells. Dev. Biol. 42, 75-83 (1975).

34. Shen, X., Yu, L., Weir, J. W. \& Gorovsky, M. A. Linker histones are not essential and affect chromatin condensation in vivo. Cell 82, 47-56 (1995).

35. HAYASHI, T., HAYASHI, H. \& IWAI, K. Tetrahymena Histone H1. Isolation and Amino Acid Sequence Lacking the Central Hydrophobic Domain Conserved in Other H1 Histones1. J. Biochem. 102, 369-376 (1987).

36. Wu, M., Allis, C. D., Richman, R., Cook, R. G. \& Gorovsky, M. A. An intervening sequence in an unusual histone H1 gene of Tetrahymena thermophila. Proc. Natl. Acad. Sci. 83, 8674-8678 (1986).

37. Dou, Y., Mizzen, C. A., Abrams, M., Allis, C. D. \& Gorovsky, M. A. Phosphorylation of linker histone H1 regulates gene expression in vivo by mimicking H1 removal. Mol. Cell 4, 641-7 (1999).

38. Dou, Y. \& Gorovsky, M. A. Phosphorylation of linker histone H1 regulates gene expression in vivo by creating a charge patch. Mol. Cell 6, 225-31 (2000).

39. Dou, Y. \& Gorovsky, M. A. Regulation of transcription by $\mathrm{H} 1$ phosphorylation in Tetrahymena is position independent and requires clustered sites. Proc. Natl. Acad. Sci. USA 99, 6142-6 (2002).

40. Wu, M. et al. Four distinct and unusual linker proteins in a mitotically dividing nucleus are derived from a 71-kilodalton polyprotein, lack p34cdc2 sites, and contain protein kinase A sites. Mol. Cell. Biol. 14, 10-20 (1994).

41. Iwamoto, M. et al. Nuclear localization signal targeting to macronucleus and micronucleus in binucleated ciliate Tetrahymena thermophila. Genes Cells 23, 568-579 (2018).

42. Roque, A., Iloro, I., Ponte, I., Arrondo, J. L. R. \& Suau, P. DNA-induced secondary structure of the carboxyl-terminal domain of histone H1. J. Biol. Chem. 280, 32141-7 (2005).

43. Teo, G. et al. SAINTexpress: improvements and additional features in Significance Analysis of INTeractome software. J. Proteomics 100, 37-43 (2014). 
44. Kataoka, K. \& Mochizuki, K. Heterochromatin aggregation during DNA elimination in Tetrahymena is facilitated by a prion-like protein. J. Cell Sci. 130, 480-489 (2017).

45. Miao, W. et al. Microarray analyses of gene expression during the Tetrahymena thermophila life cycle. PLoS One 4, e4429 (2009).

46. González-Romero, R., Eirín-López, J. M. \& Ausió, J. Evolution of high mobility group nucleosome-binding proteins and its implications for vertebrate chromatin specialization. Mol. Biol. Evol. 32, 121-31 (2015).

47. Hock, R., Furusawa, T., Ueda, T. \& Bustin, M. HMG chromosomal proteins in development and disease. Trends Cell Biol. 17, 72-9 (2007).

48. Xu, J., Tian, H., Liu, X., Wang, W. \& Liang, A. Localization and functional analysis of HmgB3p, a novel protein containing highmobility-group-box domain from Tetrahymena thermophila. Gene 526, 87-95 (2013).

49. Qiao, J., Xu, J., Bo, T. \& Wang, W. Micronucleus-specific histone H1 is required for micronuclear chromosome integrity in Tetrahymena thermophila. PLoS One 12, e0187475 (2017).

50. Valpuesta, J. M., Martín-Benito, J., Gómez-Puertas, P., Carrascosa, J. L. \& Willison, K. R. Structure and function of a protein folding machine: the eukaryotic cytosolic chaperonin CCT. FEBS Lett. 529, 11-6 (2002).

51. Mayer, M. P. \& Bukau, B. Hsp70 chaperones: cellular functions and molecular mechanism. Cell. Mol. Life Sci. 62, 670-84 (2005).

52. Campos, E. I. et al. The program for processing newly synthesized histones H3.1 and H4. Nat. Struct. Mol. Biol. 17, 1343-51 (2010).

53. Niessen, M., Schneiter, R. \& Nothiger, R. Molecular Identification of virilizer, a Gene Required for the Expression of the SexDetermining Gene Sex-lethal in Drosophila melanogaster (2001).

54. Suganuma, T., Pattenden, S. G. \& Workman, J. L. Diverse functions of WD40 repeat proteins in histone recognition. Genes Dev. 22, 1265-8 (2008)

55. Downs, J. A., Kosmidou, E., Morgan, A. \& Jackson, S. P. Suppression of homologous recombination by the Saccharomyces cerevisiae linker histone. Mol. Cell 11,1685-92 (2003).

56. Garg, J. et al. Conserved Asf1-importin $\beta$ physical interaction in growth and sexual development in the ciliate Tetrahymena thermophila. J. Proteomics 94, 311-326 (2013).

57. Nabeel-Shah, S., Ashraf, K., Pearlman, R. E. \& Fillingham, J. Molecular evolution of NASP and conserved histone H3/H4 transport pathway. BMC Evol. Biol. 14, 139 (2014).

58. Mosammaparast, N. et al. Nuclear import of histone H2A and H2B is mediated by a network of karyopherins. J. Cell Biol. 153, 251-62 (2001).

59. Shimada, M. et al. Gene-Specific H1 Eviction through a Transcriptional Activator $\rightarrow \mathrm{p} 300 \rightarrow \mathrm{NAP} 1 \rightarrow \mathrm{H} 1$ Pathway. Mol. Cell 74, 268-283.e5 (2019).

60. Thorslund, T. et al. Histone H1 couples initiation and amplification of ubiquitin signalling after DNA damage. Nature 527, 389-393 (2015).

61. Mandemaker, I. K. et al. DNA damage-induced histone H1 ubiquitylation is mediated by HUWE1 and stimulates the RNF8RNF168 pathway. Sci. Rep. 7, 15353 (2017).

62. Lu, X. et al. Linker histone $\mathrm{H} 1$ is essential for Drosophila development, the establishment of pericentric heterochromatin, and a normal polytene chromosome structure. Genes Dev. 23, 452-465 (2009).

63. Yue, Y. et al. VIRMA mediates preferential m6A mRNA methylation in 3'UTR and near stop codon and associates with alternative polyadenylation. Cell Discov. 4, 10 (2018).

64. Kim, D. I. et al. An improved smaller biotin ligase for BioID proximity labeling. Mol. Biol. Cell 27, 1188-96 (2016).

65. Fillingham, J. S., Bruno, D. \& Pearlman, R. E. Cis-acting requirements in flanking DNA for the programmed elimination of mse2.9: a common mechanism for deletion of internal eliminated sequences from the developing macronucleus of Tetrahymena thermophila. Nucleic Acids Res. 29, 488-98 (2001).

66. Fillingham, J. S. et al. Molecular genetic analysis of an SNF2/brahma-related gene in Tetrahymena thermophila suggests roles in growth and nuclear development. Eukaryot. Cell 5, 1347-59 (2006).

67. Saettone, A. et al. The bromodomain-containing protein Ibd1 links multiple chromatin-related protein complexes to highly expressed genes in Tetrahymena thermophila. Epigenetics Chromatin 11, 10 (2018).

68. Liu, G. et al. Data Independent Acquisition analysis in ProHits 4.0. J. Proteomics 149, 64-68 (2016)

69. Cline, M. S. et al. Integration of biological networks and gene expression data using Cytoscape. Nat. Protoc. 2, 2366-82 (2007).

70. Letunic, I., Doerks, T. \& Bork, P. SMART 7: recent updates to the protein domain annotation resource. Nucleic Acids Res. 40, D302-5 (2012).

71. Kumar, S., Stecher, G. \& Tamura, K. MEGA7: Molecular Evolutionary Genetics Analysis Version 7.0 for Bigger Datasets. Mol. Biol. Evol. 33, 1870-1874 (2016).

72. Yang, J. et al. The I-TASSER Suite: protein structure and function prediction. Nat. Methods 12, 7-8 (2015).

73. Buchan, D. W. A. \& Jones, D. T. The PSIPRED Protein Analysis Workbench: 20 years on. Nucleic Acids Res., https://doi.org/10.1093/ nar/gkz297 (2019).

74. Schneider, C. A., Rasband, W. S. \& Eliceiri, K. W. NIH Image to ImageJ: 25 years of image analysis. Nat. Methods 9, 671-5 (2012).

\section{Acknowledgements}

We thank Anita Samardzic for technical assistance. Work in the Fillingham and Lambert laboratories was supported by the Natural Sciences and Engineering Research Council of Canada (NSERC) Discovery Grants RGPIN-2015-06448 and RGPIN-2017-06124, respectively. The Fillingham laboratory was also supported by a grant from the Ryerson University Health Research Fund. J.-P.L. holds a Junior 1 salary award from the Fonds de Recherche du Québec-Santé (FRQ-S) and was also supported through a John R. Evans Leaders Fund from the Canada Foundation for Innovation (37454). Work in the Pearlman laboratory was supported by Canadian Institutes of Health Research (CIHR) MOP13347 and Natural Sciences and Engineering Research Council of Canada (NSERC) Discovery Grant 539509.

\section{Author contributions}

S.N.-S. designed the overall study, carried out structural, phylogenetic and expression analyses, participated in AP-MS, prepared final figures, wrote the manuscript and coordinated edits from all the authors. K.A. generated Mlh1-FZZ cell lines, performed AP-MS and IF experiments. A.S. generated Hho1-FZZ cell lines, performed AP-MS and IF analysis. J.G. participated in AP-MS. J.D. participated in Hho1-FZZ generation. J.-P.L. performed all the MS experiments, participated in data analysis and contributed to manuscript editing. R.E.P. provided reagents, participated in manuscript editing and co-supervised the project. J.F. conceived the study, participated in manuscript editing and project co-supervision.

\section{Competing interests}

The authors declare no competing interests. 


\section{Additional information}

Supplementary information is available for this paper at https://doi.org/10.1038/s41598-019-56867-0.

Correspondence and requests for materials should be addressed to R.E.P. or J.F.

Reprints and permissions information is available at www.nature.com/reprints.

Publisher's note Springer Nature remains neutral with regard to jurisdictional claims in published maps and institutional affiliations.

(c) (i) Open Access This article is licensed under a Creative Commons Attribution 4.0 International License, which permits use, sharing, adaptation, distribution and reproduction in any medium or format, as long as you give appropriate credit to the original author(s) and the source, provide a link to the Creative Commons license, and indicate if changes were made. The images or other third party material in this article are included in the article's Creative Commons license, unless indicated otherwise in a credit line to the material. If material is not included in the article's Creative Commons license and your intended use is not permitted by statutory regulation or exceeds the permitted use, you will need to obtain permission directly from the copyright holder. To view a copy of this license, visit http://creativecommons.org/licenses/by/4.0/.

(c) The Author(s) 2020 